\title{
On Supplying LEDs from Very Low de Voltages with High Frequency AC-LED Drivers
}

\author{
Ignacio Castro, Member, IEEE, Aitor Vazquez, Member, IEEE, Daniel G. Aller, Student Member, IEEE, Manuel \\ Arias, Member, IEEE, Diego G. Lamar, Member, IEEE, and Javier Sebastian, Senior Member, IEEE. \\ e-mail: gams.castro@gmail.com
}

\begin{abstract}
This paper studies the driving of white Light Emitting Diodes (LEDs) from very low voltages in the 1.2 to $2 \mathrm{~V}$ range. The proposed idea is based on replacing the standard Schottky diode used in conventional converter topologies (i.e., buck, buck-boost and boost) with an LED while shortcutting the output of the converter. In this configuration, the LED works both as the load and the rectifier diode of the converter, hence switching the LED at high frequencies $(>100 \mathrm{kHz}$ ). Moreover, a thorough analytical study is carried out for the two topologies proposed in this paper, emphasizing in particular their static analysis and the obtaining of the boundaries between the different conduction modes. Finally, the idea is experimentally validated by means of the boost converter variation (i.e., the DL//S AC-LED driver). The DL//S ACLED driver was also compared with a dc-dc boost converter, showing a better luminous efficacy while disposing of the Schottky diode and the output capacitor. The analysis was carried out when connected to a Li-Ion battery using a simple control and integrated circuit for its development.
\end{abstract}

Keywords- AC-LED driving, LED driver, low input voltages and single-cell battery.

\section{INTRODUCTION}

Light Emitting Diodes (LEDs) are increasingly becoming the main source of artificial light in our homes, offices and streets due to their reliability, long life, energy efficiency and low maintenance requirements. It is a well-known fact that LEDs can only be driven by forward currents, as they behave like a diode, allowing the current to flow only in one direction. This fact has made the driving of LEDs from AC grids a major research topic in the search to replace old, inefficient luminaries [1]-[3]. In fact, if the current source is not acceptable for the driving of the luminaire, a dc-dc converter needs to adapt the level of current to drive the LED accurately.

For the application under study, which is that of driving a single LED from a very low voltage (i.e., a battery cell whose voltage can vary in the 1.2 to $2 \mathrm{~V}$ range), the dc-dc converter needs to be able to boost the input voltage to a suitable value. This voltage needs to be higher than the knee-voltage of the LED, which is usually around $3 \mathrm{~V}$, for it to start illuminating. Previous literature considers the Joule Thief to be the preferred solution for driving LEDs from very low voltages, based on a blocking oscillator circuit [4], [5], due to its low component

This work was supported in part by the Spanish Government under Project MINECO-17-DPI2016-75760-R, in part by the Principality of Asturias under Severo Ochoa grant BP14-142, Project FC-15-GRUPIN14-143 and Project SVPA-17-RIS3-4, and in part by European Regional Development Fund (ERDF) grants. count and simple driving. However, the lack of ease in implementing a current feedback loop and the low efficiency displayed (below 80\%) are the main drawbacks of this solution. In order to solve these issues, a conventional dc-dc converter can be suitable for this task based on either a boost, see Fig. 1 (a), or a buck-boost converter, see Fig. 1 (c). The use of these topologies is currently a possibility at the low input voltage of the application due to the newer Pulse Width Modulation (PWM) driving solutions based on micro-power dc-dc converters, which, in the case of the boost converter, are able to achieve roughly $88 \%$ efficiency at their peak. However, the losses resulting from the voltage drop of a conventional PN diode (typically, from 1 to $1.5 \mathrm{~V}$ ) will cause the electrical efficiency to plummet. Moreover, using a Schottky diode or active rectification is not an option due to the cost restrictions of the application. In fact, even when using a Schottky diode, the voltage drop will still be significant (typically from 0.25 to 0.5 $\mathrm{V})$. Therefore, what is proposed in this paper is to replace the rectifier diode on traditional dc-dc converters with a white LED while short-circuiting the output of the converter. As a result, two different converters can be obtained: an LED paralleled with a Switch (a DL//S AC-LED driver; see Fig. 1 (b)) and an LED paralleled with an Inductor (a DL//L AC-LED driver; see Fig. 1 (d). Note that while the LEDs in conventional dc-dc converters are supplied with a constant current, the LED in the DL//S and DL//L AC-LED drivers is supplied with a pulsed current, pulsing at the same frequency at which the main switch $(\mathrm{S})$ is operating. The operation of the LED at high frequencies $(>100$ $\mathrm{kHz}$ ) acting as the regular diode of a power converter, also referred to in the literature as high-frequency AC-LED driving, has been studied by means of resonant dual half bridges [6]-[8], a flyback converter working in Discontinuous Conduction Mode (DCM) [9], self-oscillating topologies [10] and quasi-resonant converters [11], [12]. All these studies conclude that LEDs are able to switch at high frequencies under different current waveforms. The latest papers perform a reliability analysis which shows that this method can provide a long lifetime for the LEDs as long as the reverse recovery effect is avoided in them [11], [12].

This paper explores the possibility of using LEDs as the rectifier diodes of dc-dc converters with the help of the two proposed LED drivers, seeking to avoid the reverse recovery effect by operating in DCM without incurring in increasing the complexity of the LED driver [13], [14]. Furthermore, an analytical study is carried out for both topologies in Section II to 

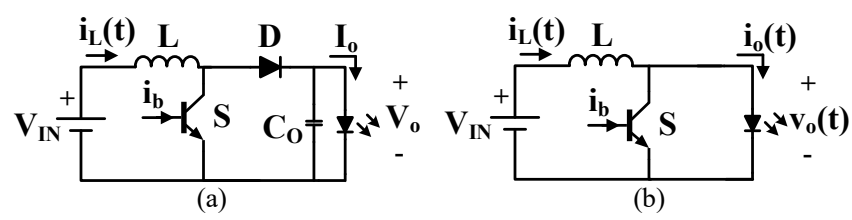

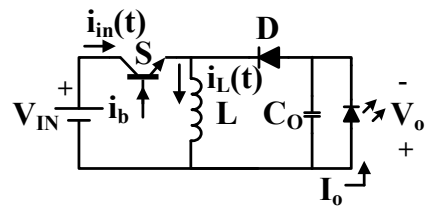

(c)

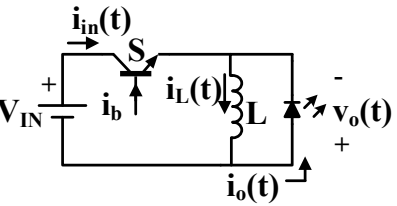

(d)
Fig. 1. Basic converters. (a) Boost converter. (b) DL//S AC-LED driver. (c) Buck-boost converter. (d) DL//L AC-LED driver.

understand their advantages and limitations, as well as to obtain their analytical waveforms. The analysis is subsequently completed by studying and normalizing the boundaries between Continuous Conduction Mode (CCM) and DCM for both converters. Section III discusses the proposed control methodology based on the use of a simple IC. In order to elucidate which is the best solution, an experimental comparison is performed in Section IV in terms of luminous efficacy between the conventional topologies and the proposed topologies in the different operating modes (CCM and DCM). Finally, the most important conclusions drawn from this study are discussed in Section V.

\section{WORKING PRINCIPLE}

As in conventional dc-dc converters, the proposed topologies are based on a PWM signal that drives the main switch (S), causing the inductance to magnetize or demagnetize in accordance with a given duty cycle (d) that turns the main switch on and off. Taking into account this principle, there are three equivalent circuits for each topology: 1) Transistor conducting, 2) Diode conducting, and 3) Neither of them conducting. The last stage will only appear when operating in DCM, which will be the aim of this study; hence the importance of the last subsection studying the boundaries between CCM and DCM.

\section{A. Static Analysis of the DL//S AC-LED Driver}

From this basic principle, there are three possible equivalent circuits for the DL//S AC-LED driver. These are shown in Fig. 2 considering the equivalent circuit of the LED as the series connection of an ideal diode, an equivalent dynamic resistance $\left(\mathrm{r}_{\text {LED }}\right)$, and an ideal voltage source $\left(\mathrm{V}_{\gamma_{-} \text {LED }}\right)$.

The magnetizing stage, see Fig. 2 (a), refers to the period of time when the main switch is conducting (i.e., $\mathrm{t}<\mathrm{dT}_{\mathrm{s}}$ ), hence linearly charging the inductance in accordance with

$$
i_{L}(t)=i_{L_{\text {valley }}}+\frac{V_{I N}}{L} t,
$$

where $\mathrm{V}_{\mathrm{IN}}$ is the input voltage, $\mathrm{L}$ is the inductance value, and $\mathrm{i}_{\mathrm{L}_{-} \text {valley }}$ corresponds to the minimum current through the inductance, as can be seen in Fig. 3 (a). Consequently, during this stage, there is no current across the LED. The end of this stage is marked by the turn-off of the main switch, which will

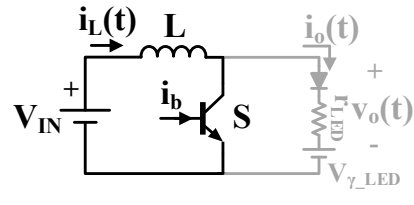

(a)

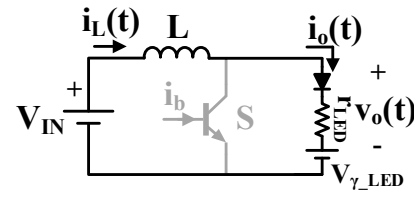

(b)

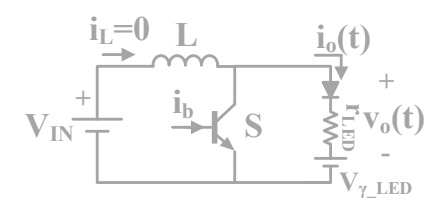

(c)

Fig. 2. Equivalent circuits for the DL//S topology. (a) Conduction of the switch. (b) Conduction of the LED (c) No conduction of either the switch or the LED.

stop conducting, causing $\mathrm{L}$ to start demagnetizing through the LED, see Fig. 2 (b).

The demagnetizing stage, see Fig. 2 (b), refers to the period when the inductance demagnetizes as the LED starts conducting. This effect is represented in the current by

$$
i_{L}(t)=i_{L_{-} a s y m}+\left(i_{L_{-} p e a k}-i_{L_{-} a s y m}\right) e^{-\frac{\left(t-d \cdot T_{S}\right)}{\tau}}
$$

where $T_{S}$ is the switching period and $i_{L_{-} \text {asym }}$ is the asymptotic value that the curve tends towards, as shown in Fig. 3 (a), $\tau$ is the time constant, and $i_{L \_ \text {peak }}$ is the maximum value of $i_{L}(t)$. The values taken by $\mathrm{i}_{\mathrm{L}_{-} \text {asym }}$ and $\tau$ are:

$$
i_{L_{-} a s y m}=\frac{V_{I N}-V_{\gamma_{-} L E D}}{r_{L E D}},
$$

and,

$$
\tau=\frac{L}{r_{L E D}} .
$$

Note that the current does not demagnetize linearly in this stage as it does in conventional converters, due to the lack of an output capacitor behaving as a constant voltage source. Consequently, it depends on the value of $r_{\text {LED }}$.

The duration of this stage is completely reliant on the operation mode of the converter (either CCM or DCM). Under CCM operation, its duration will be determined by the duty cycle of the main switch, the duration being $(1-\mathrm{d}) \mathrm{T}_{\mathrm{s}}$; whereas for DCM operation, its duration will be marked by $i_{L}(t)$ reaching the zero current value.

For CCM operation, the following condition needs to be fulfilled in the steady state:

$$
i_{L}\left(T_{S}\right)=i_{L_{-} \text {valley }}
$$

Then, on the basis of this assumption, it is possible to obtain the values of $i_{L_{-} \text {valley }}$ and $i_{L \_p e a k}$ as

$$
i_{L_{-} \text {valley }}=\frac{i_{L_{-} \text {asym }}\left(1-e^{\frac{(d-1) T_{S}}{\tau}}\right)+\frac{V_{I N} d T_{S}}{L} e^{\frac{(d-1) T_{S}}{\tau}}}{1-e^{\frac{(d-1) T_{S}}{\tau}}},
$$

and 


$$
i_{L_{-} \text {peak }}=i_{L_{-} \text {valley }}+\frac{V_{I N}}{L} d T_{S} .
$$

Equations (3), (4), (6) and (7) provide (1) and (2) with the required parameters in order to obtain the expressions of $i_{L}(t)$ and $\mathrm{i}_{\mathrm{o}}(\mathrm{t})$ during a switching period. It then becomes possible to obtain the average power delivered to the LED within a switching period by integrating the instantaneous power consumed by the LED as

$$
P_{L E D}=\frac{1}{T_{s}} \int_{d T_{S}}^{T_{S}}\left(i_{L}(t) V_{\gamma_{-} L E D}+i_{L}(t)^{2} r_{L E D}\right) d t,
$$

where $i_{L}(t)$ is defined by $(2)$.

The no conduction stage, see Fig. 2 (c), is an optional stage that occurs under DCM operation considering that there is no energy left in the inductance to continue driving the LED. The duration of this stage can be defined as the time during which the current through the inductance is zero, until the main switch is turned on again, see Fig. 3 (b).

For operation in DCM, the study is quite similar to that carried out for CCM; hence, (1)-(4) and (7) are valid for this mode. In this scenario, the assumption made in the steady state is:

$$
i_{L_{-} \text {valley }}=0 .
$$

Note that in DCM, (2) is only valid during the time interval $\mathrm{d}^{\prime} \mathrm{T}_{\mathrm{s}}$, which is defined as the time during which the current through the LED is higher than zero, see Fig. 3 (b). Consequently, d' can be calculated by substituting (9) in (2) and solving for d', yielding

$$
d^{\prime}=\frac{\tau}{T_{S}} \cdot \ln \left(\frac{i_{L_{-} p e a k}-i_{L_{-} a s y m}}{-i_{L_{-} a s y m}}\right) .
$$

At this point, the average power delivered to the LED can be obtained in a similar way to CCM operation as

$$
P_{L E D}=\frac{1}{T_{S}} \int_{d T_{S}}^{\left(d^{\prime}+d\right) T_{S}}\left(i_{L}(t) V_{\gamma_{-} L E D}+i_{L}(t)^{2} r_{L E D}\right) d t .
$$

The boundary between CCM and DCM can be studied by matching d' in (10) to $1-d$. Then,

$$
1-d=\frac{L}{T_{S} \cdot r_{L E D}} \cdot \ln \left[\frac{d T_{s} \frac{V_{I N}}{L}-i_{L_{-} a s y m}}{-i_{L_{-} a s y m}}\right]
$$

However, (12) happens to be a transcendental equation, which means that numerical methods are required to study the boundary between the two modes. Considering the difficulties resulting from the above statement, a normalized analysis is carried out employing some base magnitudes so as to be able to graphically analyze the conduction mode of the LED driver.

\section{B. Normalized Analysis of the DL//S AC-LED Driver}

In order to normalize the study of the DL//S AC-LED driver, it is mandatory to select the base magnitudes to normalize, see Table I. These base magnitudes can then be used to define the following normalized expressions:

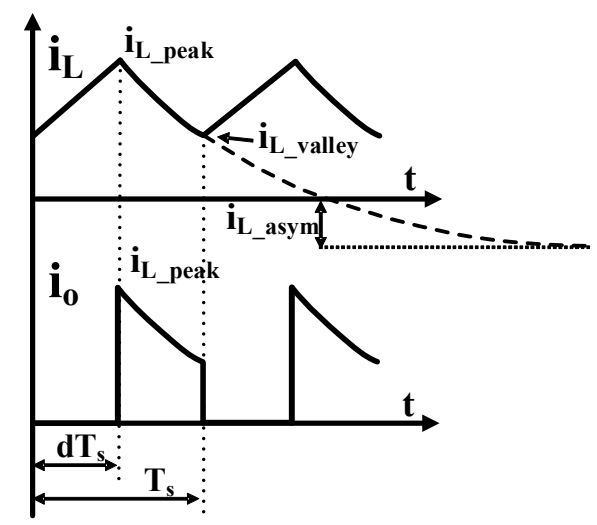

(a)

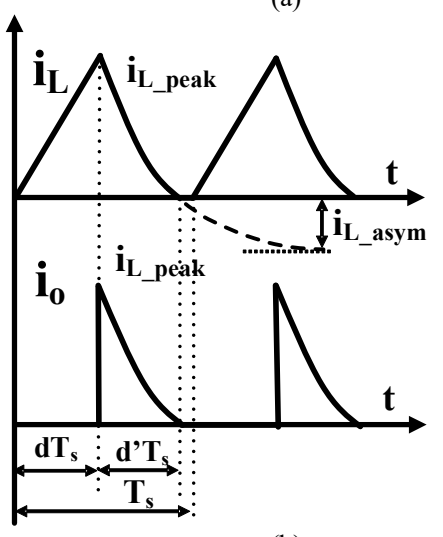

\begin{tabular}{|c|c|}
\hline Unit & BASE VALUE \\
\hline Time & $\mathrm{T}_{\mathrm{s}}$ \\
\hline Voltage & $\mathrm{V}_{\gamma_{-} \text {LED }}$ \\
\hline Impedance & $r_{L E D}$ \\
\hline Current & $\mathrm{I}_{\text {base }}=\mathrm{V}_{\gamma_{L} \mathrm{LED}} / \mathrm{r}_{\mathrm{LED}}$ \\
\hline Power & $P_{\text {base }}=\left(V_{\gamma_{-} L E D}\right)^{2} / r_{\text {LED }}$ \\
\hline & \\
\hline \multirow{2}{*}{\multicolumn{2}{|c|}{$\begin{array}{c}\tau_{n}=\frac{\tau}{T_{S}}=\frac{L}{r_{L E D} T_{S}} \\
V_{G n}=\frac{V_{I N}}{V_{\gamma_{-} L E D}},\end{array}$}} \\
\hline & \\
\hline$i_{L n}(t)=$ & $\frac{i_{L}(t) r_{L E D}}{V_{\gamma_{-} L E D}}$ \\
\hline$L_{-}$valleyn $=$ & $\frac{i_{L_{-} \text {valley }} r_{L E D}}{V_{\gamma_{-} L E D}}$, \\
\hline
\end{tabular}

(b)

Fig. 3. Most representative waveforms for the DL//S AC-LED driver. (a) CCM (b) DCM.

TABLE I. VALUES OF THE BASE MAGNITUDES USED IN THE NORMALIZATION 


$$
\begin{gathered}
i_{L_{-} \text {peakn }}=\frac{i_{L_{\perp} \text { peak }}}{I_{\text {base }}}=\frac{i_{L_{\_} \text {peak }} r_{L E D}}{V_{\gamma_{-} L E D}}, \\
P_{L E D n}=\frac{P_{L E D}}{P_{\text {base }}}=\frac{P_{L E D} r_{L E D}}{\left(V_{\gamma_{-} L E D}\right)^{2}} .
\end{gathered}
$$

At this point, (3) and (14) can be used to rewrite (12) as:

$$
d V_{I N n}+\tau_{n}\left(1-V_{G n}\right)\left(1-e^{\frac{1-d}{\tau_{n}}}\right)=0 .
$$

Equation (20) depends on numerous parameters. However, most of them are well-known in the design process, such as, $\mathrm{V}_{\gamma_{L} \text { LED }}, \mathrm{r}_{\mathrm{LED}}$, and $\mathrm{V}_{\mathrm{IN}}$. Therefore, it is only necessary to study the boundary between CCM and DCM by varying $\tau_{\mathrm{n}}$ and $\mathrm{d}$. To this end, Fig. 4 represents the value of $d$ on the boundary between modes ( $d_{\text {crit }}$ ) for different $V_{G n}$ and $\tau_{n}$, see Fig. 4 (a), and for the critical $\tau_{n}$ value $\left(\tau_{\text {ncrit }}\right.$ ) for different values of $V_{G n}$ and $d$, see Fig. 4 (b). The importance of Fig. 4 is such that it helps the designer select the desired operating mode, in accordance with the following conditions:

- For operation in CCM, either $d$ needs to be greater than $\mathrm{d}_{\text {crit }}$ for given values of $\mathrm{V}_{\mathrm{Gn}}$ and $\tau_{\mathrm{n}}$, as in Fig. 4 (a), or $\tau_{\mathrm{n}}$ needs to be greater than $\tau_{\text {ncrit }}$ for given values of $d$ and $\mathrm{V}_{\mathrm{Gn}}$, as in Fig. 4 (b).

- For operation in DCM, either $d$ needs to be lower than $\mathrm{d}_{\text {crit }}$ for given values of $\mathrm{V}_{\mathrm{Gn}}$ and $\tau_{\mathrm{n}}$, as in Fig. 4 (a), or $\tau_{\mathrm{n}}$ needs to be lower than $\tau_{\text {ncrit }}$ for given values of $d$ and $\mathrm{V}_{\mathrm{Gn}}$, as in Fig. 4 (b).

Continuing the normalization process, the normalized value of the average $\mathrm{P}_{\mathrm{LED}}, \mathrm{P}_{\mathrm{LEDn}}$, can be obtained using (13)-(19). In this particular case, (8) and (11) can be unified within a single expression given that the only difference between the two is the expression of the upper limit of the integral. Therefore,

$$
P_{L E D n}=\int_{d}^{d^{\prime}+d}\left(i_{L n}(t)+i_{L n}(t)^{2}\right) d t_{n} .
$$

where d' takes the following values depending on its operating mode:

$$
\begin{gathered}
C C M: d^{\prime}=1-d, \\
D C M: d^{\prime}=\tau_{n} \ln \left[\frac{\frac{V_{G n} d}{\tau_{n}}+1-V_{G n}}{1-V_{G n}}\right] .
\end{gathered}
$$

As regards $i_{\operatorname{Ln}}(t)$, which is obtained by normalizing (2) with the help of (7), its expression considering DCM operation (i.e., $i_{L_{-} \text {valley }}=0$ ) can be defined by:

$$
\begin{aligned}
i_{L n}(t)=V_{G n}-1 & +\left(i_{L_{-} \text {valleyn }}-V_{G n}+1\right. \\
& \left.+\frac{d V_{G n}}{\tau_{n}}\right) e^{\frac{\left(d-t_{n}\right)}{\tau_{n}}},
\end{aligned}
$$

where $\mathrm{i}_{\mathrm{L}_{-} \text {valleyn }}$ takes the following values for CCM and DCM operation:

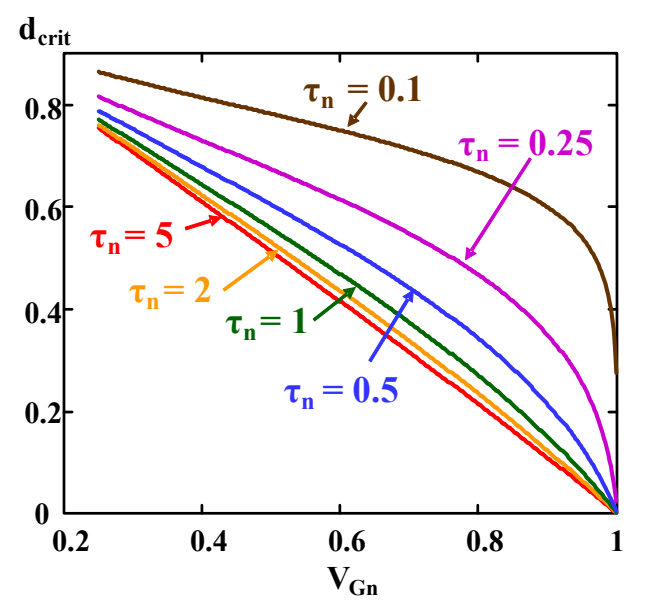

(a)

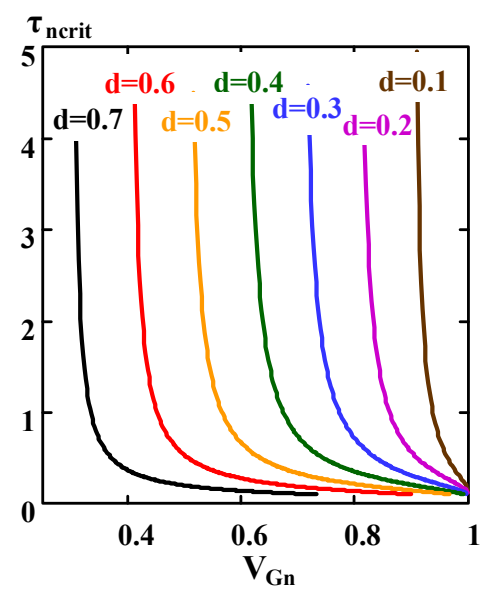

(b)

Fig. 4. Boundary between conduction modes for the DL//S AC-LED driver. (a) $d_{\text {crit }}$ versus $V_{G n}$ and $\tau_{n}$. (b) $\tau_{n}$ versus $d$ and $V_{G n}$.

$$
\begin{gathered}
C C M: i_{L_{-} \text {valleyn }} \\
=\frac{V_{G n}-1+\left(-V_{G n}+1+\frac{d V_{G n}}{\tau_{n}}\right) e^{\frac{(d-1)}{\tau_{n}}}}{1-e^{\frac{(d-1)}{\tau_{n}}}}, \\
D C M: i_{L_{-} \text {valleyn }}=0 .
\end{gathered}
$$

At this point of the analysis, it is now possible to study the variation of $P_{L E D n}$ when varying $V_{G n}, d$ and $\tau_{n}$. Fig. 5 represents this variation, showing that the power given to the LED drastically increases when the DL//S AC-LED driver changes its operating mode from DCM to CCM. It can also be seen that the power transferred to the LED is completely reliant on the parameter $\tau_{\mathrm{n}}$ under DCM operation, becoming inconsequential under CCM. This behaviour is identical to any dc-dc converter, where the dc gain depends on $\mathrm{d}$ for $\mathrm{CCM}$ operation, whereas for DCM it depends on several parameters such as: $\mathrm{L}, \mathrm{T}_{\mathrm{s}}$, and the load. From these statements, it can be seen that $\tau_{\mathrm{n}}$ plays the same role as the dimensionless parameters $\mathrm{k}$ (i.e., $\mathrm{k}=2 \mathrm{~L} /\left(\mathrm{RT}_{\mathrm{s}}\right)$ ) in conventional dc-de converters [15]. 

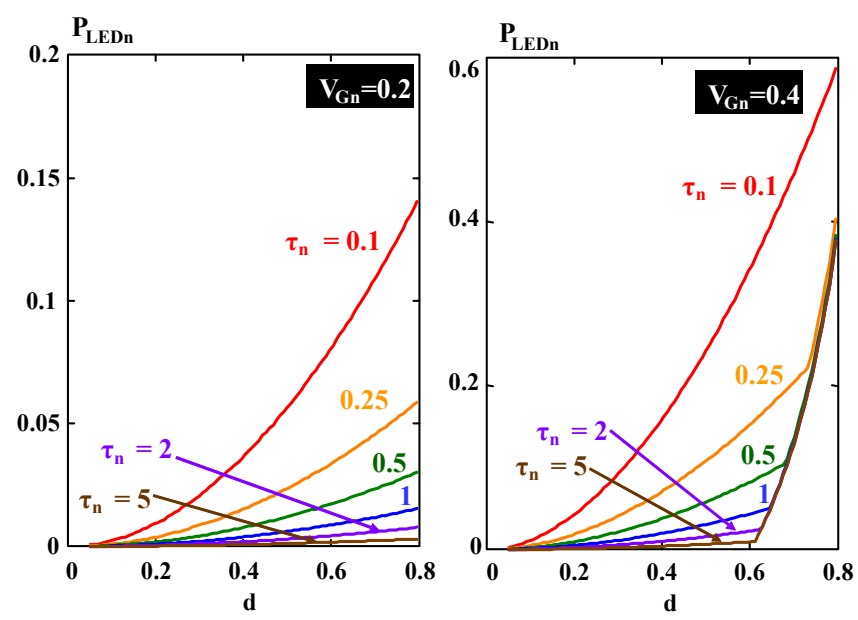

Fig. 5. Normalized average power consumed by the LED in the

\section{Static Analysis of the DL//L AC-LED Driver}

The analysis carried out for the DL//L AC-LED driver is quite similar to that of the DL//S AC-LED driver, as it has the same three equivalent circuits, which are depicted in Fig. 6. The most significant change appears in $\mathrm{i}_{\mathrm{L}_{\text {asym}}}$, which, for this ACLED driver, happens to be:

$$
i_{L_{-} a s y m}=\frac{-V_{\gamma_{L} L E D}}{r_{L E D}}=-I_{\text {base }} .
$$

Consequently, the only equations that change from the previous analysis are (20), (23), (24) and (25), obtaining:

$$
\begin{gathered}
d V_{G n}+\tau_{n}\left(1-e^{\frac{1-d}{\tau_{n}}}\right)=0, \\
d^{\prime}=\tau_{n} \ln \left[\frac{d V_{G n}}{\tau_{n}}+1\right], \\
i_{L n}(t)=-1+\left(i_{L_{-} \text {valleyn }}+1+\frac{d \cdot V_{G n}}{\tau_{n}}\right) e^{\frac{\left(d-t_{n}\right)}{\tau_{n}}},
\end{gathered}
$$

and

$$
i_{L_{-} \text {valleyn }}=\frac{\left(1+\frac{d V_{G n}}{\tau_{n}}\right) \cdot e^{\frac{(d-1)}{\tau_{n}}}-1}{1-e^{\frac{(d-1)}{\tau_{n}}}} .
$$

These expressions complete the analysis of the DL/L AC-LED driver, it being possible to perform the same graphical representations as those made for the DL//S AC-LED driver in order to study the boundary between conduction modes and the average power transferred by the LED driver to the LED.

The boundary between modes is studied in Fig. 7. Note that the statements introduced for the DL//S AC-LED driver are still valid for this LED driver. In addition, it can be seen in Fig. 7 (a) that the variation of $\tau_{\mathrm{n}}$ does not affect the boundary as much as in the previous converter.

Finally, for the DL//L AC-LED driver, the power transferred to the LED is studied in Fig. 8 for different values of $V_{G n}, d$ and $\tau_{\mathrm{n}}$. If these results are compared to those in Fig. 5, it can be seen that the performance of this converter is extremely similar, but it is unable to transfer the same amount of power to the LED for the same values of $\mathrm{V}_{\mathrm{Gn}}, \mathrm{d}$, and $\tau_{\mathrm{n}}$.

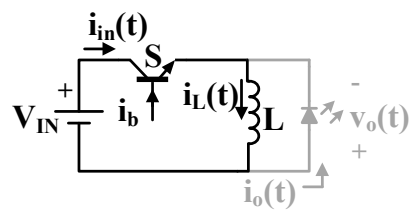

(a)

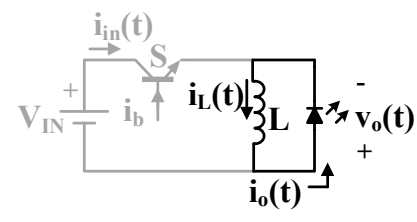

(b)

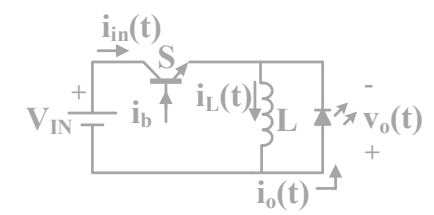

(c)

Fig. 6. Equivalent circuits for the DL/L AC-LED driver. (a) Conduction of the switch. (b) Conduction of the LED (c) No conduction of either switch or LED.

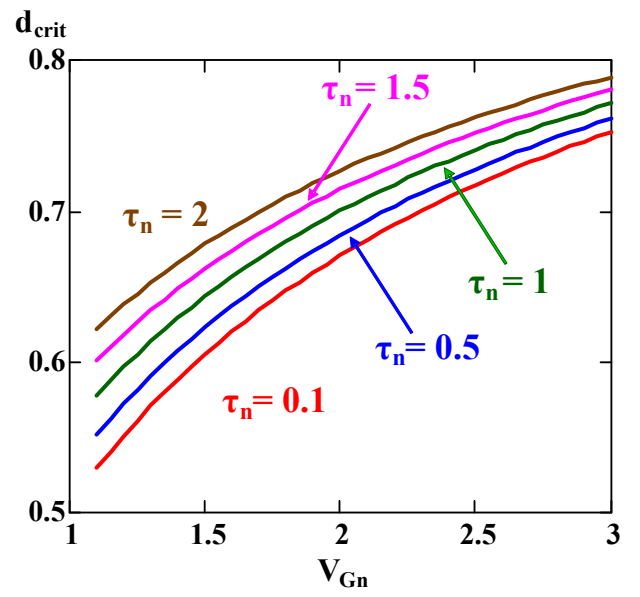

(a)

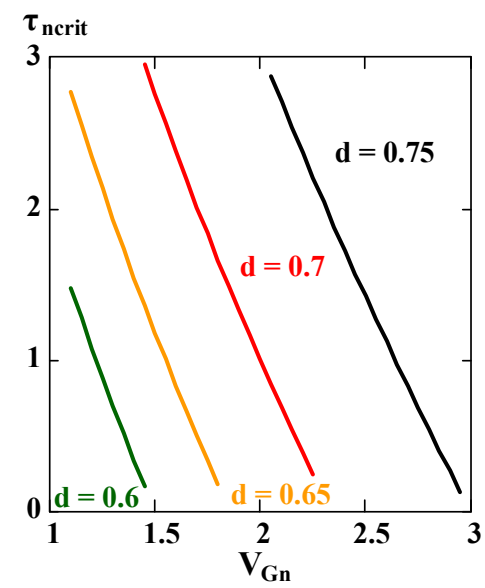

(b)

Fig. 7. Boundary between conduction modes for the DL//L LED driver. (a) $d_{\text {crit }}$ versus $V_{G n}$ and $\tau_{n}$. (b) $\tau_{n}$ versus $d$ and $V_{G n}$. 

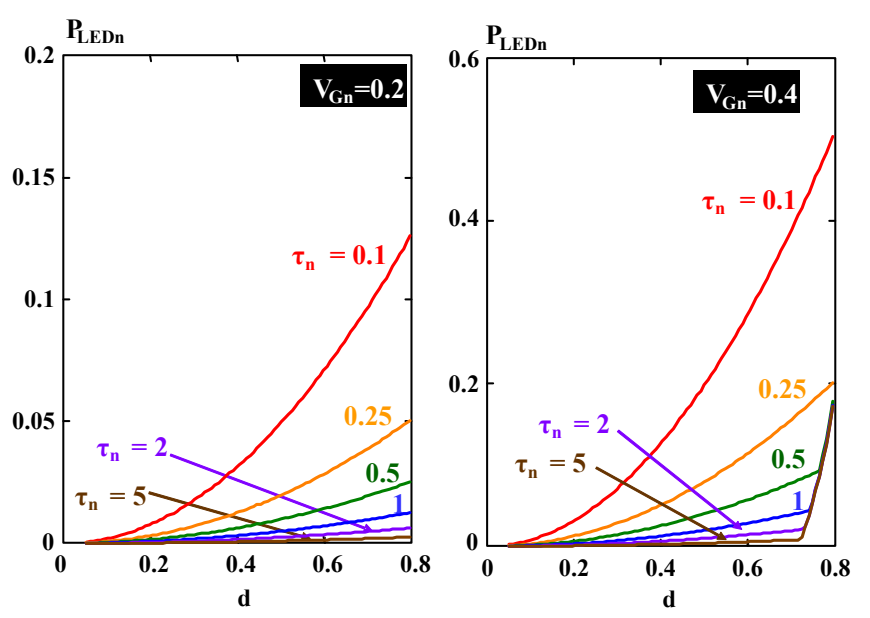

Fig. 8. Normalized average power consumed by the LED in the DL//L LED driver for different values of $V_{G n}, d$ and $\tau_{n}$.

\section{Proposed Control}

One of the key features of using a dc-dc converter to drive LEDs is the ability to control the current supplied to the LEDs using a feedback control loop. In the particular case of the proposed AC-LED drivers, the use of a feedback control loop would require filtering the high frequency current that feeds the LEDs. This would mean adding more components to the design, which is undesirable. However, the aim of this study is actually to control the peak current value on the switch, just like in a peak current mode control (PCMC) [15].

Fig. 9 shows the proposed control particularized for the DL//S AC-LED driver using the LT1308B. As can be seen, the current across the main switch is measured and a compensation ramp is added. Then, this voltage is compared to a voltage reference $\left(\mathrm{V}_{\text {ref }}\right)$ that sets the peak value of the current. By varying $\mathrm{V}_{\text {ref, }}$, by means of a potentiometer it is possible to achieve dimming on the LED, given that, by controlling the peak current, the duty cycle on the main switch is also being controlled. In addition to its simplicity, the use of a PCMC provides not only the ability to control the current across the LEDs, but it also inherently protects both the main switch and the LED, as it limits the maximum peak current on both semiconductors.

The previous paragraphs develop the idea of controlling the converter with a well-known and massively used control methodology. The other problem that remains is to be able to provide enough voltage from such a low input without penalizing the efficiency of the LED driver. Fortunately, this problem has been solved for single-cell Li-Ion chargers, usually by using the boosted output voltage as a means to drive the required components [17]. Therefore, there are a handful of integrated circuits that can be used for this task, such as the one that will be used in the next section and that has been introduced in Fig. 9, namely the LT1308B [18].

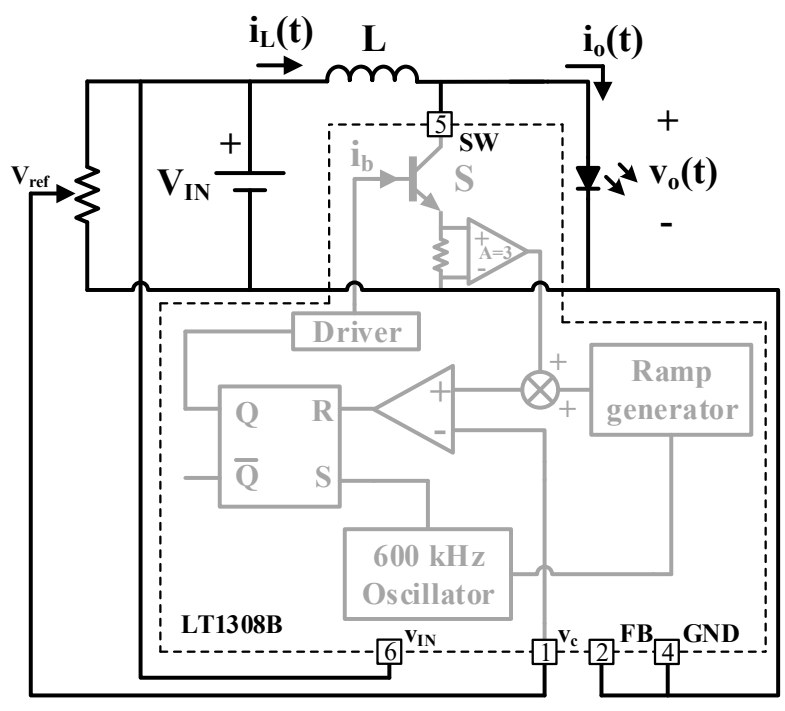

Fig. 9. Proposed control for the AC-LED drivers under study, particularized for the DL//S AC-LED driver with the LT1308B.

\section{EXPERIMENTAL RESULTS}

In order to validate the analysis introduced in the previous section, two prototypes of the DL//S AC-LED driver were designed and experimentally tested. The first prototype was designed to validate the analysis in terms of operating modes, whereas the second was designed with the aim of achieving a compact final design using a Li-Ion battery as its main source and including the proposed control, see Fig. 10. The first prototype works in the 1 to $2 \mathrm{~V}$ range, switching at $100 \mathrm{kHz}$ and feeding a W42180T LED $\left(\mathrm{V}_{\gamma_{L} L E D}=2.8 \mathrm{~V}\right.$ and $\left.\mathrm{r}_{\mathrm{LED}}=1.2 \Omega\right)$. The first analysis is carried out in terms of the boundaries between CCM and DCM. Therefore, for $\mathrm{d}$ equal to 0.5 and $\tau_{\mathrm{n}}$ equal to 1 , Fig. 11 shows the transition between modes when the input voltage of the DL//S AC-LED driver varies. In fact, by using Fig. 4 (a) and considering the aforementioned $\mathrm{d}$ and $\tau_{\mathrm{n}}$, it is simple to observe that, for a value of $\mathrm{V}_{\mathrm{Gn}}$ below 0.6, the driver should work in DCM. This value coincides with the experimental results, as Fig. 11 (a) shows the correct operation in DCM for $\mathrm{V}_{\mathrm{Gn}}$ equal to 0.43 , while Fig. 11 (b) shows the operation at a point considered close to critical conduction mode for $\mathrm{V}_{\mathrm{Gn}}$ equal to 0.57 .

In order to complete the analysis of operating modes, Fig. 12 shows the variation of $\tau_{\mathrm{n}}$ for fixed values of $\mathrm{V}_{\mathrm{Gn}}$ and $\mathrm{d}$. It correctly demonstrates the expected performance shown in Fig. 4 (b), in which, for $\mathrm{d}$ equal to 0.5 at a $\mathrm{V}_{\mathrm{Gn}}$ of 0.57 , those values of $\tau_{\mathrm{n}}$ below 1 make the AC-LED driver operate in DCM. In contrast, values of $\tau_{\mathrm{n}}$ higher than one make the AC-LED driver operate in CCM. Furthermore, Fig. 13 shows the operation of the DL//S AC-LED driver for different values of $d$ while keeping the other two parameters constant.

It should be noted that the operation in CCM causes the reverse recovery phenomenon to occur [13], as can be observed in Fig. 11 (c), Fig. 12 (b), and Fig. 13 (b). In contrast, DCM operation does not show this effect due to the zero current switching towards the turn-off. However, this operation in DCM will limit the maximum power provided to the LED due to the 


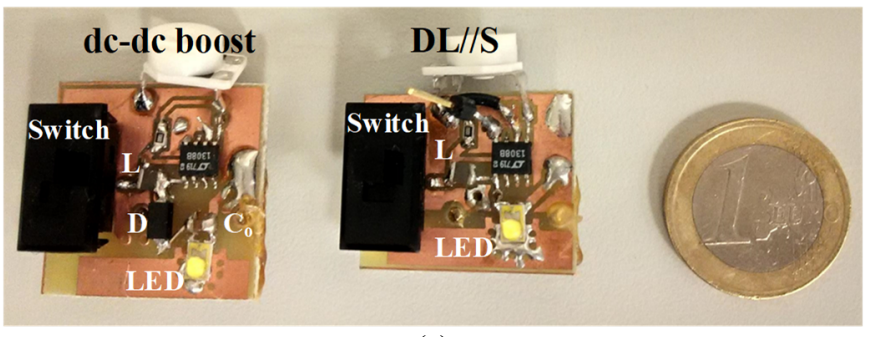

(a)

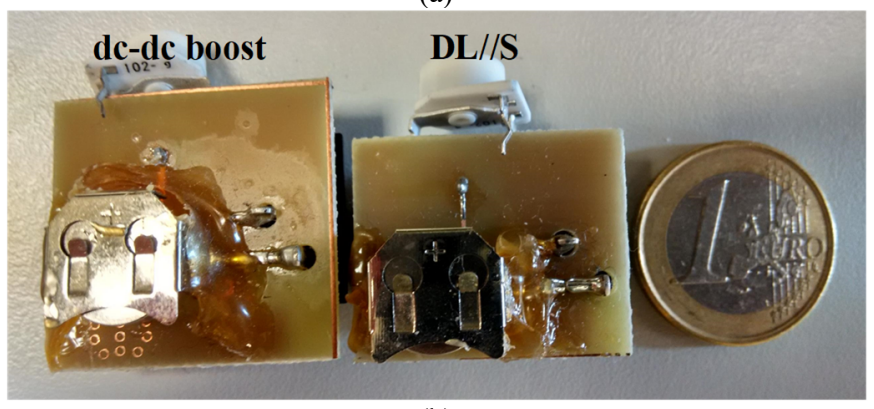

(b)

Fig. 10. Experimental prototypes of the conventional dc-dc LED boost and DL//S AC-LED driver. (a) Top layer. (b) Bottom layer.

higher peak current when compared with the same average current in CCM. Moreover, as already stated, DCM operation is not as efficient in terms of the amount of power transferred to the LED.

Nonetheless, as studied in [11] and [12], the reverse recovery effect affects LEDs negatively, influencing their lifetime, which is why this effect needs to be removed. The aforementioned papers specifically addressed its removal by slightly increasing the complexity of the control stage and adding an inductance. Precisely for the case under study, cost and practicality are of the utmost importance, which is why operation in DCM is attractive, even if it may limit the efficiency or the maximum output power of the AC-LED driver.

For all the above reasons, the second prototype was built with the aim of minimizing its size, being designed to operate in boundary conduction mode at maximum power. In fact, in order to reduce the stress of the peak current across the LED, the duty cycle is maximized by selecting it as the first duty curve that crosses the line of $\mathrm{V}_{\mathrm{Gn}}$ under nominal conditions in Fig. 4 (b). It is then compared to a conventional dc-dc boost converter LED driver, which is also shown in Fig. 5. The characteristics of the DL//S AC-LED driver and the conventional dc-dc boost LED driver are similar, with an input voltage varying from 1 to $2 \mathrm{~V}$, a switching frequency of $600 \mathrm{kHz}$, an inductance of $1.2 \mu \mathrm{H}$, and driving an LXML-PWC2 LED $\left(\mathrm{V}_{\gamma_{-} \text {LED }}=2.6 \mathrm{~V}\right.$ and $\mathrm{r}_{\mathrm{LED}}=$ $0.5 \Omega$ ). The main difference between the prototypes is that the conventional dc-dc boost LED driver requires the use of an ultralow forward voltage Schottky diode (UPS115Ue3), whose cost is similar to that of the LED, and an output capacitor. These two elements would significantly increase the cost of this solution, as both converters will require the same IC to perform the driving from very low input voltages (i.e., the LT1308B).

It should also be noted that in Fig. 10 an external analog switch is added to be able to turn on and off the converter without requiring to change the potentiometer level. In addition,

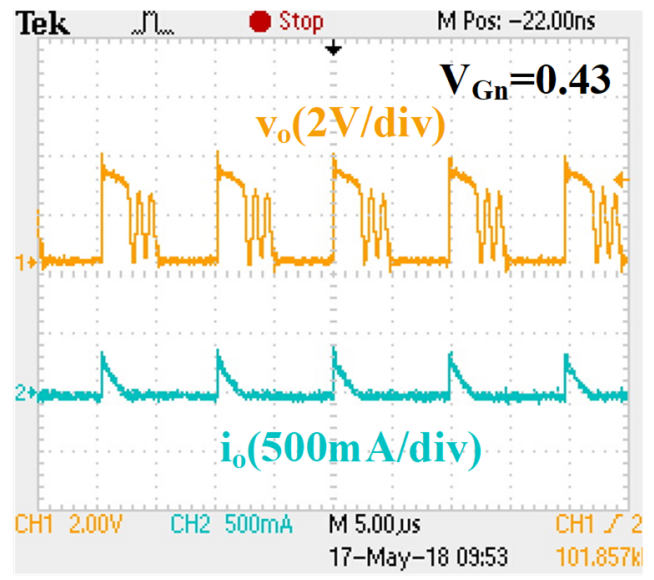

(a)

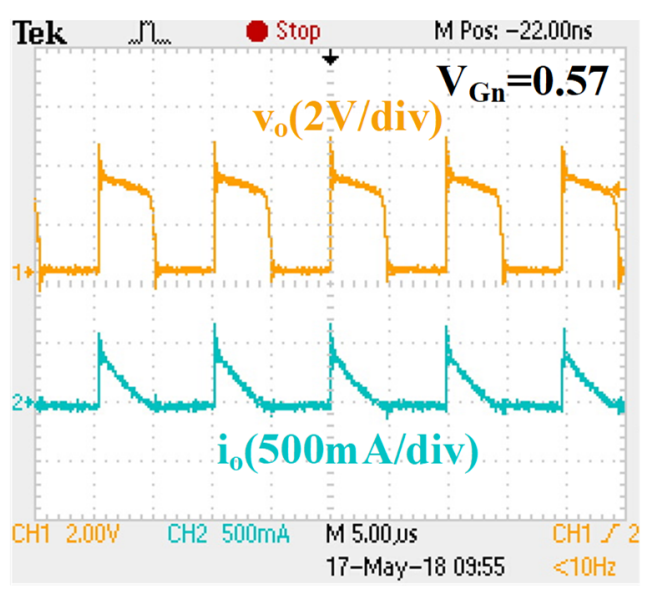

(b)

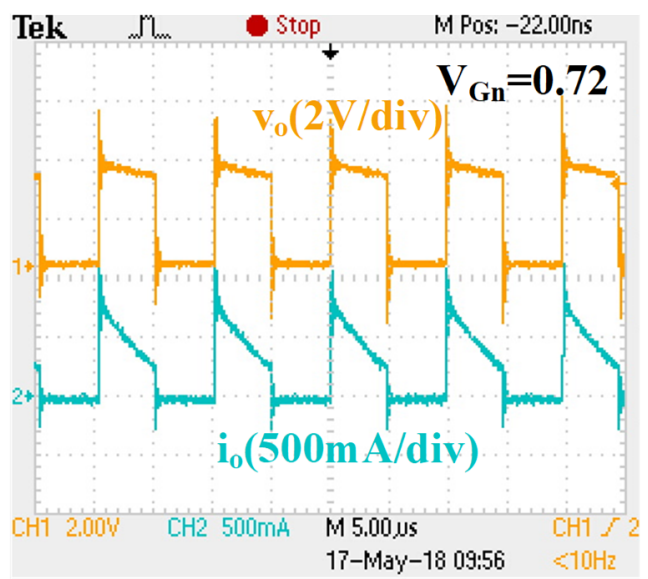

(c)

Fig. 11. Experimental waveforms of the DL//S AC-LED driver for different values of $\mathrm{V}_{\mathrm{Gn}}$ while keeping $\mathrm{d}=0.5$ and $\tau_{\mathrm{n}}=1$. (a) $\mathrm{V}_{\mathrm{Gn}}=0.43$. (b) $\mathrm{V}_{\mathrm{Gn}}=0.57$. (c) $\mathrm{V}_{\mathrm{Gn}}=0.72$.

both prototypes are compared in terms of size in this figure, however the disposal of the Schottky diode and the output capacitor is not as significant in size as it is in terms of cost.

In order to carry out a fair comparison between the DL//S AC-LED driver and the conventional dc-dc boost LED driver, 


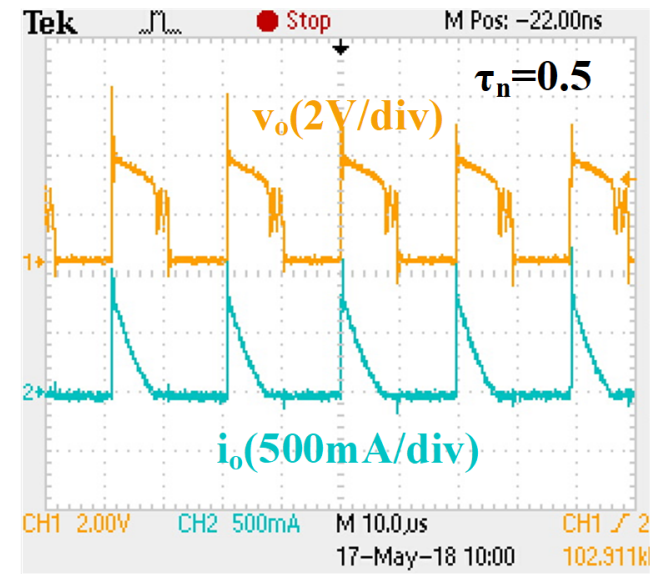

(a)

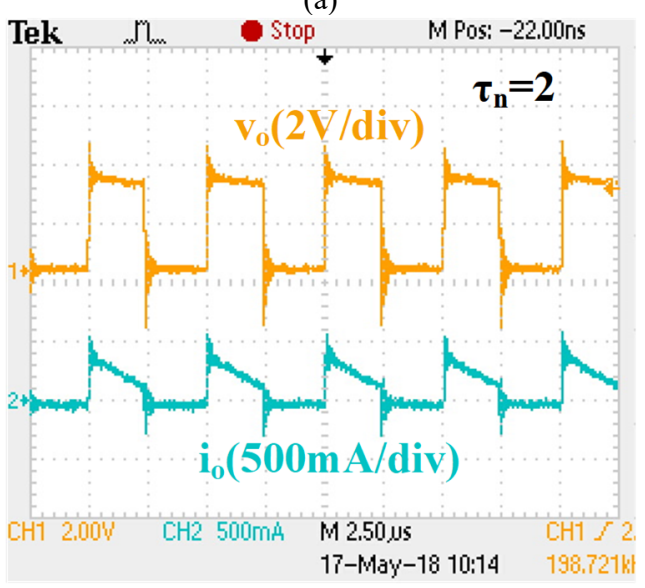

(b)

Fig. 12. Experimental waveforms of the DL//S AC-LED driver for different values of $\tau_{n}$ while keeping $d=0.5$ and $V_{G n}=0.57$. (a) $\tau_{n}=0.5$. (b) $\tau_{\mathrm{n}}=2$.

Fig. 14 shows the measured luminous efficacy measured for both LED drivers when varying the output power while keeping $\mathrm{V}_{\mathrm{Gn}}$ and $\tau_{\mathrm{n}}$ constant at 0.57 and 1.44 , respectively. The variation of the output power is done with a potentiometer than changes the peak current condition across the LED and the main switch, as stated in Section III, changing at the same time the average current across the LED string and being able to perform full dimming. In addition, the luminous flux is measured within an integrated sphere with the help of a light to volt converter based on a photodiode connected to a transimpedance amplifier (namely, a TSL-257), and a spectrocolorimeter from LabSphere Inc., and then accordingly converted to $\mathrm{lm} / \mathrm{W}$. As can be seen, the luminous efficacy is similar for the lower output power values and becomes higher for the proposed topology at the higher output power values due to the voltage drop of the rectifier diode and losses. It is important to note that, even though, a triangular current waveform across the LEDs is theoretically not as efficient as dc current driving [19] due to the losses on the Schottky diode, this a priori disadvantage in terms of driving methodology is completely overcome by the proposed solution. This validates not only the cost efficiency of the proposed AC-LED driver, but also offers an advantage in terms of the light output per watt.

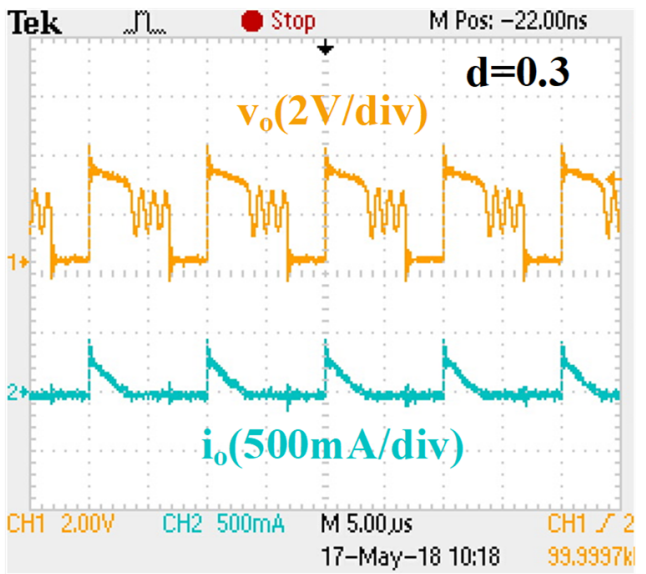

(a)

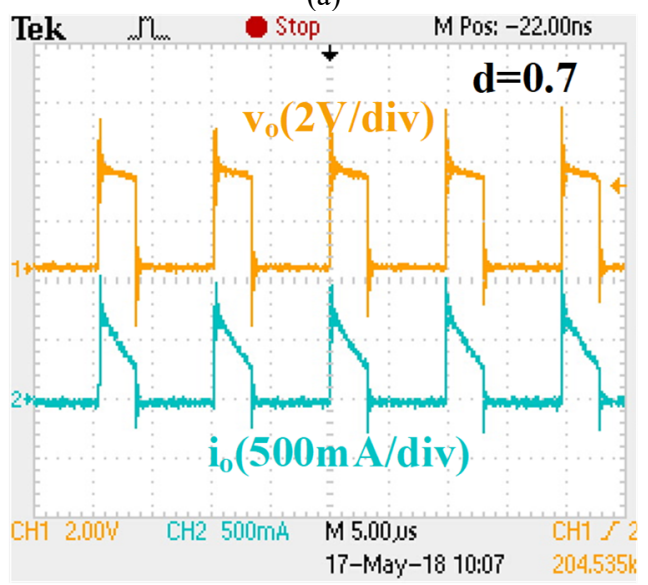

(b)

Fig. 13. Experimental waveforms of the DL//S AC-LED driver for different values of $d$ while keeping $\tau_{n}=1$ and $V_{G n}=0.57$. (a) $d=0.3$.

(b) $d=0.7$.

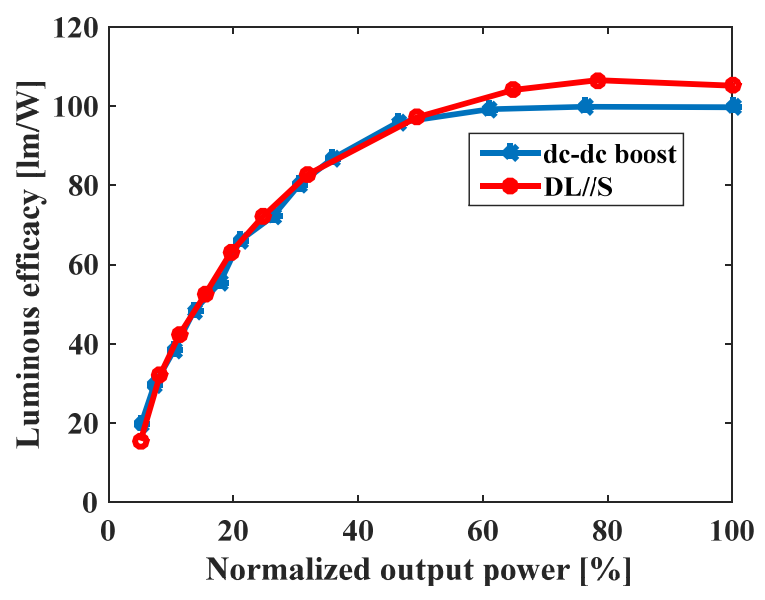

Fig. 14. Luminous efficacy versus normalized output power for the dc-dc boost LED driver and the DL//S AC-LED driver.

As regards the DL//L AC-LED driver, the experimental analysis carried out for the DL//S AC-LED driver is quite similar in terms of understanding the boundaries between modes and its design. The only comment that should be added with respect to its design is the fact that the LEDs will need to withstand negative voltages. Although this is undesirable, given that this 
voltage never surpasses the knee-voltage, it is safe to consider its operation in accordance with [20]. Nonetheless, it will still depend on the characteristics set by the manufacturer for the selected LED.

\section{CONCLUSIONS}

This paper addresses the driving of LEDs from very low voltages by using them as the rectifier of conventional dc-dc converters, becoming an AC-LED driver. The analysis carried out in this study renders two new topologies; one of them has been experimentally tested and compared to a traditional dc-dc boost converter with a peak efficiency at an input voltage of 1.5 $\mathrm{V}$ of $85 \%$. The proposed DL//S AC-LED driver shows a better performance in terms of luminous efficacy while reducing the cost of a conventional dc-dc converter that disposes of the Schottky diode and the output capacitance. The proposed control based on current programmed control is simple, protects both the main switch and the LED, and reduces the amount of components required, as it is already included within the selected IC. Furthermore, the selected IC solves the main problem of driving an LED from very low voltages, as it already includes all the necessary circuitry to generate the auxiliary voltages to drive the main switch of the LED driver, thus being able to drive a single LED from a single $1.2 \mathrm{~V} \mathrm{Li-Ion} \mathrm{battery.}$

\section{REFERENCES}

[1] M. Arias, A. Vazquez, and J. Sebastian, "An overview of the AC-DC and DC-DC converters for LED lighting applications," Automatika-J. Control, Measure., Electr., Comput. Commun., vol. 53, pp. 156-172, 2012.

[2] Y. Chen, C. Chang and P. Yang, "A novel constant current control circuit for a primary-side controlled AC-DC LED driver," 2014 11th International Conference on Electronics, Computer and Computation (ICECCO), Abuja, 2014, pp. 1-4.

[3] J. Yang, A. N. Faris, W. Zhang, Y. Liu and X. Chen. "A universal-input high-power-factor LLC resonant driver without electrolytic capacitor for PWM dimming LED lighting application," 2014 International Power Electronics and Application Conference and Exposition, Shanghai, 2014, pp. 1473-1478.

[4] John E. Bohan, Jr., "Low voltage driven oscillator circuit", U.S. patent 4734 658A, March 29, 1988.

[5] Z. Kaparnik, "One Volt LED - A Bright Light", Everyday Practical Electronics (EPE) magazine, November 1999.

[6] B. G. Kang, Y. Choi and S. K. Chung, "High frequency AC-LED driving for street light," 2015 9th International Conference on Power Electronics and ECCE Asia (ICPEECCE Asia), Seoul, 2015, pp. 1246-1251.

[7] C. L. Kuo, T. J. Liang, K. H. Chen and J. F. Chen, "Design and implementation of high frequency AC-LED driver with digital dimming," Proceedings of 2010 IEEE International Symposium on Circuits and Systems, Paris, 2010, pp. 3713-3716.

[8] K. H. Loo, Y. M. Lai and C. K. Tse, "Design and Analysis of LCC Resonant Network for Quasi-Lossless Current
Balancing in Multistring AC-LED Array," in IEEE Transactions on Power Electronics, vol. 28, no. 2, pp. 1047-1059, Feb. 2013.

[9] J. C. W. Lam and P. K. Jain, "A High Power Factor, Electrolytic Capacitor-Less AC-Input LED Driver Topology With High Frequency Pulsating Output Current," in IEEE Transactions on Power Electronics, vol. 30, no. 2, pp. 943-955, Feb. 2015.

[10] M. A. Juárez, P. R. Martínez, G. Vázquez, J. M. Sosa and M. Ponce, "Analysis and design for self-oscillating LED driver with high frequency pulsating output current," IECON 2015 - 41st Annual Conference of the IEEE Industrial Electronics Society, Yokohama, 2015, pp. 003992-003996.

[11] I. Castro, S. Lopez, K. Martin, M. Arias, D. G. Lamar and J. Sebastian, "High frequency dc-dc AC-LED driver based on ZCS-QRCs," 2017 IEEE Energy Conversion Congress and Exposition (ECCE), Cincinnati, OH, 2017, pp. 36883695.

[12] I. Castro, S. Lopez, K. Martin, M. Arias, D. G. Lamar and J. Sebastian, "A family of high frequency AC-LED drivers based on ZCS-QRCs," in IEEE Transactions on Power Electronics, vol. PP, no. 99, pp. 1-1.

[13] P. Haaf, and J. Harper. "Understanding diode reverse recovery and its effect on switching losses.", 2007.

[14] M. M. Jovanovic, "A technique for reducing rectifier reverse-recovery-related losses in high-power boost converters," in IEEE Transactions on Power Electronics, vol. 13, no. 5, pp. 932-941, Sep 1998.

[15] S. Cuk and R. D. Middlebrook, "A general unified approach to modelling switching DC-tO-DC converters in discontinuous conduction mode," 1977 IEEE Power Electronics Specialists Conference, Palo Alto, CA, USA, 1977, pp. 36-57.

[16] R. Redl and N. O. Sokal, "Current-mode control, five different types, used with the three basic classes of power converters: Small-signal AC and large-signal DC characterization, stability requirements, and implementation of practical circuits," 1985 IEEE Power Electronics Specialists Conference, Toulouse, France, 1985, pp. 771-785.

[17] R. K. Williams, B. Mohandes and Chae Lee, "Highfrequency $\mathrm{DC} / \mathrm{DC}$ converter for lithium-ion battery applications utilizes ultra-fast $\mathrm{CBiC} / \mathrm{D}$ process technology," Applied Power Electronics Conference and Exposition, 1995. APEC '95. Conference Proceedings 1995., Tenth Annual, Dallas, TX, 1995, pp. 322-332 vol.1.

[18] "High Current, Micropower Single Cell, 600kHz DC/DC Converters", Linear Technology, Linear Technology, 2 pp. 1996, 97 Linear Technology.

[Online] http://www.analog.com/media/en/technicaldocumentation/data-sheets/1308abfb.pdf

[19] W. Feng, Y. He and F. G. Shi, "Investigation of LED Light Output Performance Characteristics Under Different Alternating Current Regulation Modes," in IEEE Journal 
of Selected Topics in Quantum Electronics, vol. 17, no. 3, pp. 720-723, May-June 2011.

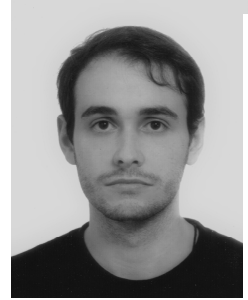

Ignacio Castro (S'14, M'18) was born in Gijón, Spain, in 1989.

$\mathrm{He}$ received the M.Sc. degree in telecommunication engineering and the $\mathrm{Ph} . \mathrm{D}$. in electrical engineering from the University of Oviedo, Gijón, Spain, in 2014 and 2018, respectively.

From 2014 to 2018, he has been a research assistant and member of the power supply group in the University of Oviedo.

In August 2018, he joined United Technologies Research Centre in Ireland as a Senior Research Engineer.

His research interests include low and medium power dc$\mathrm{dc}$ converters, modeling, simulation and control of $\mathrm{dc}-\mathrm{dc}$ converters and power-factor correction ac-dc converters.

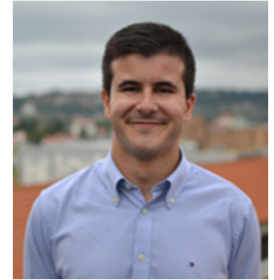

Aitor Vazquez (S'2010, M'2015) was born in Oviedo, Spain, in 1984. He received the M.Sc. degree in telecommunication engineering, in 2009 , and the $\mathrm{PhD}$. degree in electrical engineering in 2016, from the University of Oviedo, Gijon, Spain.

He became a Member of the Power Supply System Group, University of Oviedo, in 2010. His current research interests include modularization techniques applied

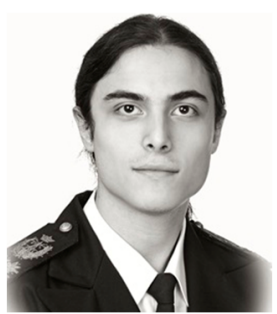

Daniel G. Aller (S'16) was born in Oviedo, Spain, in 1992. He received the M. Sc. degree in Telecommunication engineering in 2016 from the University of Oviedo, Gijon, Spain, where he is currently working towards the Ph.D degree in electrical engineering.

His research interests include LED drivers for visible light communication, high-frequency DC-DC converters and wide bandgap semiconductors.
[20] "Cree ${ }^{\circledR}$ XLamp® LED Electrical Overstress," Appl. Note CLD-AP29 REV 1E, Cree, Inc., Durham, NC, USA, 2016.

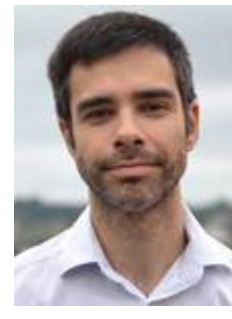

Manuel Arias (S'05 M'10) received the M. Sc. degree in electrical engineering from the University of Oviedo, Spain, in 2005 and the $\mathrm{Ph}$. D. degree from the same university in 2010 .

In 2007 he joined the University of Oviedo as an Assistant Professor and since 2016 he is an Associate Professor at the same university.

His research interests include ac-dc and dc-dc power converters, battery-cell equalizers and LED lighting.

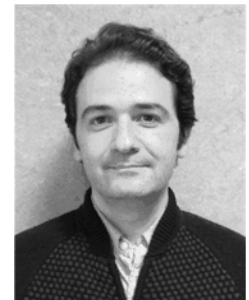

Diego G. Lamar (S'05-M'08) was born in Zaragoza, Spain, in 1974. He received the M.Sc. and Ph.D. degrees in electrical engineering from the Universidad de Oviedo, Gijón, Spain, in 2003 and 2008, respectively.

In 2003 and 2005 he became a Research Engineer and an Assistant Professor respectively at the University of Oviedo. Since September 2011, he has been an Associate Professor. His research interests are focused in switching-mode power supplies, converter modelling, and power-factor-correction converters.

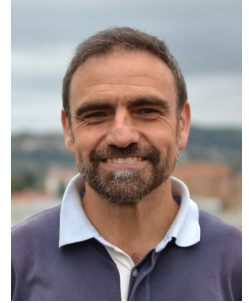

Javier Sebastián (M'87-SM'11) was born in Madrid, Spain, in 1958. He received the M.Sc. degree from the Technical University of Madrid (UPM), and the Ph.D. degree in electrical engineering from the University of Oviedo, Spain, in 1981 and 1985, respectively.

He was an Assistant Professor and an Associate Professor at both the UPM and the University of Oviedo. Since 1992, he has been with the University of Oviedo, where he is currently a Professor. His research interests are switching-mode power supplies, modeling of dc-to-dc converters, low output voltage dc-to-dc converters, high power factor rectifiers, LED drivers, dc-to-dc converters for envelope tracking techniques and the use of wide band-gap semiconductors in power supplies. 